\title{
La simulación en el mundo del trabajo, Colombia 1911-1957
}

\author{
Óscar Gallo 1 \\ Sandra Caponi ${ }^{2}$ \\ María Fernanda Vásquez 3
}

\section{Resumen}

La Ley 57 de 1915, dispuso que fueran los médicos los encargados de evaluar la magnitud de los accidentes de trabajo. Además de determinar el tiempo de incapacidad y la indemnización, ellos tenían la función de revelar en el examen de ingreso las constituciones mórbidas o predisposiciones a la accidentalidad, y los casos de "simulación" después del accidente de trabajo. En este artículo, se analiza la forma como los médicos del trabajo objetivaron la simulación en Colombia. También se puede apreciar que la forma como la psiquiatría francesa definió y clasificó la simulación, tuvo eco en la mayoría de los médicos colombianos, pero en la práctica médicolegal la dinámica económica determinó, en buena medida, los alcances de la discusión. Aunque no es un problema exclusivo del mundo del trabajo, si es este un escenario ideal para observar la construcción social de la enfermedad.

Palabras-clave: Simulación. Psiquiatría. Medicina Legal. Accidentes de Trabajo. Enfermedades Profesionales. Seguridad Social.

\section{Introducción}

En 1953, el abogado cartagenero Eloy Tous Liñán publicó el artículo Interés médico-legal de los accidentes de trabajo. Tous, considera que la unión de la ciencia médica con la ciencia del derecho ayuda a hacer más justo y eficaz el derecho social. Piensa además que es delicada y digna de verdaderos especialistas la fijación de las incapacidades del trabajo, entre otras razones, porque existe un tipo común de post-accidentados llamados simuladores, "que por fraude o engaño aparentan algo que en realidad no tienen, demuestran anomalías o trastornos psíquicos que no existen". Para Tous, la

\footnotetext{
1 Doctor en Historia de la Universidad Federal de Santa Catarina. Actualmente se desempeña como Investigador de la Escuela Nacional Sindical (Colombia) y como docente de la Universidad Eafit. E-mail: oscargallovelez@gmail.com.

2 Professora do Departamento de Sociologia e Ciências Políticas. Universidad Federal de Santa Catarina. Pesquisadora de CNPq. E-mail: sandracaponi@gmail.com.

3 Doutora em Ciências Humanas. Pós-Doutoranda do Programa Interdisciplinar em Ciências Humanas (PPGICH), Universidade Federal de Santa Catarina. Bolsista PNPD-CAPES. E-mail: mfdavasquez@gmail.com.
} 
facilidad con que el "juzgador" puede ser engañado por este tipo de individuos se debe a que no está obligado a profundizar en apreciaciones psicológicas (TOUS LIÑÁN, 1953, p. 45).

En principio, persiguiendo una alusión directa de Tous a José Ingenieros se tenía la idea de que los clásicos La simulación en la lucha por la vida (1900) y La simulación de la locura (1903), habían sido decisivos en la forma como los médicos colombianos se aproximaron a la cuestión.

En efecto, este psiquiatra argentino había cimentado su fama de prestigioso alienista, sociólogo y criminalista en los mencionados trabajos. Sus tesis sobre simulación se difundieron en América Latina, a veces de forma explícita, como es el caso de Brasil (BRUNO, 1941), y otras de manera implícita en la recuperación de muchos de sus argumentos.

Pese a que la simulación en Ingenieros estaba lejos de ser una cuestión laboral, en su trabajo insinúa varios de los elementos que tienen especial interés para el médico del trabajo. Estos son: determinar si oculta alguna enfermedad; si hay incompatibilidad entre la enfermedad y las funciones que debe desempeñar el individuo afectado; si la enfermedad, lesión o defecto físico fueron provocados. De igual forma, Ingenieros menciona como razones habituales del simulador: eludir el servicio militar; explotar la beneficencia pública y la caridad; con la finalidad de ser considerados sin responsabilidad penal para poder escapar de las penas;entrar en una corporación o establecimiento; ganar los beneficios de un seguro de vida. En conclusión, Ingenieros vislumbraba la importancia de las simulaciones de estados patológicos en la industria.

Ahora bien, no se puede olvidar que el objetivo del libro era la simulación de la locura y, concretamente,el principio de utilidad que envuelve la práctica de la simulación, la sobresimulación y la disimulación en el ámbito penal. Ingenieros estaba interesado en "la simulación de la locura con el propósito de ser considerado irresponsable y exento de pena"(INGENIEROS, 1903, p. 161). Considera que "la ventaja del delincuente simulador consiste en ser declarado irresponsable, para "curar" enseguida de su falsa locura y recuperar la libertad" (INGENIEROS, 1903, p. 161).

En el fondo, lo que incomodaba a Ingenieros ya varios contemporâneos, era la desaparición del libre albedrío, "fundamento interminable de la responsabilidad moral del criminal", que la Escuela Positivista Italiana gradualmente sustituyó por el concepto de peligrosidad (FERLA, 2009, p. 347). 
Cabe señalar, que en este estudio dedicado a la simulación en el mundo del trabajo, no existen referencias explícitas a la obra de Ingenieros aunque varias de sus contribuciones, como por ejemplo, la distinción entre diversas formas de simulación, parecen estar presentes en nuestros autores cuando se trata de analizar cuestiones limítrofes entre medicina laboral y criminología.

Descartada la pista de Tous, observamos que la simulación hacía parte del repertorio discursivo de la medicina legal desde los siglos XVI-XVIII, cuando médicos y juristas debatieron desde el punto de vista técnico y moral, las razones por las cuales los vagabundos simulaban o inventaban enfermedades para ganar dinero (FERREIRA, 2007, p. 78; PASTORE, 1999). Tal interés por la simulación se mantuvo en el siglo XIX, pasando a ser uno de los aspectos más importantes en el proceso de configuración del saber psiquiátrico. Saber quién miente o finge sobre la enfermedad y la locura, es la base de prácticamente todas las discusiones decimonónicas en el campo psiquiátrico (FOUCAULT, 2007).

Aun cuando la simulación era un problema con una larga historia en la cultura y la medicina, la pregunta por la simulación en el mundo del trabajo únicamente se planteó en Occidente a finales del siglo XIX. Esto se debea que las leyes de compensación de accidentes de trabajo surgen en este lado del mundo entre finales del siglo XIX y a comienzos del siglo XX. Pero además, porque es en ese mismo periodo que la medicina se legitimó como campo de saber encargado de determinar las razones de la muerte y el infortunio en el mundo del trabajo. En otras palabras, leyes de accidentes y medicina legal son contemporáneas y correlativas, la pregunta por la simulación emerge en este contexto a consecuencia de la histórica sospecha de la burguesía sobre la mala fe de la clase obrera.

De acuerdo con lo anterior, para analizar la cuestión de la simulación es necesario comenzar por recordar algunos aspectos sobre la medicina legal en Colombia. El artículo 65 de la Ley 100 de 1892, ordenaba que en los lugares donde había médicos oficiales "el juez o funcionario instructor, en vez de nombrar peritos [legos]", daría aviso a los médicos para que practicaran las diligencias necesarias y dieran su dictamen por escrito. Aunque el proceso de medicalización del poder judicial se había iniciado en Colombia en la segunda mitad del siglo XIX, fue con la Ley 100 que se dio el primer paso en el reconocimiento de la experticia médicolegal. En principio el papel de los médicos era ilustrar a la justicia con el conocimiento científico, pero paulatinamente se produce un "relevo de positividades" en el que los enunciados jurídicos se imbrican cada vez más con los enunciados médicos y la pericia 
médica se torna una prueba decisivaen el juicio. Finalmente en 1914, la Ley 53 estableció el Servicio Nacional de Medicina Legal en un esfuerzo infructuoso por implementar el servicio médico legal en todo El territorio nacional (DEL VALLE, 2010).

Un año después, se dictó la ley 57 sobre accidentes de trabajo. El legislador consideró oportuno que fueran los jueces municipales los encargados de tramitar los procesos. Dada la capilaridad de la justicia penal, se esperaba garantizar que los patrones y los trabajadores pudieran reportar el accidente en las 24 horas siguientes, y resolver el pleito de manera rápida.

La doctrina del riesgo que inspiraba la legislación de accidentes, presumía la responsabilidad del patrón para que el peso de la prueba no recayera en el trabajador. Sin embargo, la lógica procesal fue en el sentido contrario fijando tres instancias o documentos de prueba: certificado del patrón o el jefe de la cuadrilla; certificado médico de la empresa o el médico forense del distrito; testimonio de tres testigos. Con el certificado de comprobación del accidente, el médico debía expedir también el certificado de cura y el certificado de consolidación (MORALES, 1937, p. 56). En las empresas grandes, la emisión de estos documentos era atributodel departamento sanitario o el médico de la empresa. El trabajador tenía la opción de impugnar el concepto o solicitar otra opinión médica. Cuando la empresa no tenía doctor se solicitaba la pericia al médico oficial o cualquier otro presente en la circunscripción territorial.

De esa forma, los certificados médicos se convirtieron en el dispositivo de evaluación de las capacidades e incapacidades y la principal forma de restringir, o en el mejor de los casos facilitar, el acceso de las personas al trabajo. El médico tenía la función de revelar las constituciones mórbidas y las predisposiciones sicofisiológicas; el trabajador, por su parte, debía estar preparado para simular o disimular la enfermedad a la hora de ingresar o exagerar el accidente en el momento de ser indemnizado. Respecto de este juego de la verdad, Tirso Quintero (1949) ironiza que al obrero es inútil interrogarlo por las enfermedades de la familia, pues todos sus miembros murieron octogenarios y en muy buenas condiciones de salud.

Para algunos médicos, la finalidad de su trabajo era simplemente buscar en los parámetros previamente establecidos por la ley la equivalencia entre lesión y compensación. Para otros, esa visión sintética del acto pericial estaba lejos de la realidad, ya que para determinar la invalidez social se debía conocer con precisión las diferentes facetas del factor humano: observación sistemática de los 
riesgos, accidentes y enfermedades en diferentes sectores industriales; identificación de las predisposiciones individuales a la accidentalidad y la enfermedad; conocimiento de las necesidades psicotécnicas de cada sector industrial en función de una evaluación precisa de la magnitud de la invalidez; oferta y demanda del mercado laboral; tecnologías médicas para la ortopedia y rehabilitación del trabajador ${ }^{4}$. En síntesis, la experiencia médico legal en casos de accidentes de trabajo y enfermedades profesionales era muy diferente a la de otros campos médicolegales.

Cada uno de los aspectos enunciados implicaba la definición de los horizontes teóricos y la formulación o apropiación de conceptos útiles en términos prácticos y teóricos. Precisamente, uno de esos conceptos fue la simulación.

En la primera parte del artículo, se indaga por las filiaciones teóricas y definen las fronteras semánticas de la simulación. La segunda parte, se observa la manera cómo esa retórica científica fue apropiada por los médicos del trabajo en Colombia. En la práctica médicolegal el ambiguo concepto de la simulación chocó con las necesidades prácticas de los médicos legales y de las compañías de seguros de vida. En ese contexto, el concepto y clasificación de la simulación fue resignificado en función de la economía de la salud y de los seguros sociales, tornándose un problema médico, ideológico y económico.

Si nos restringimos al marco legal deberíamos definir un periodo temporal que abarca desde el año 1915, momento de promulgación de la primera ley de accidentes de trabajo, hasta el año 1946, fecha de formulación de la ley de accidentes que fijó la tabla de evaluación de incapacidades e indemnizaciones. Pero como nuestro objetivo aquí es analizar el problema de la simulación, el recorte temporal está directamente vinculado con las fuentes consultadas, esto es 1911 y 1957. Cualquiera de estas fechas puede parecer arbitraria, porque la simulación no es un problema exclusivo de las primeras décadas del siglo, más bien se trata de una cuestión de mucha

${ }^{4}$ En los años 1940 los médicos del trabajo se manifestaron sobre la necesidad de que las pericias de accidentes y enfermedades profesionales fueran realizadas por médicos especialistas o adjuntos a la Dirección Nacional de Higiene Industrial. En efecto, el esfuerzo por legitimar el campo de la medicina del trabajo se hizo visible en la formulación del decreto no. 841 de 1946, que establecía la tabla de enfermedades profesionales. Para su elaboración, el gobierno convocó a la Academia Nacional de Medicina. El resultado fue bastante cuestionado, pues según los especialistas, no estaba basado en ningún criterio epidemiológico, no tenía correlación alguna con las necesidades de la industria y buena parte de las enfermedades no había sido verificada en el país. Resumiendo, los académicos privilegiaron una visión clínica del problema y sin consultar expertos en medicina del trabajo copiaron códigos de otros países. 
actualidad cuya larga historia en el mundo del trabajo se dispersa en todo el siglo XX5.

En ese sentido, se analizaron dieztextos de médicos colombianos que dedicaron algunas páginas o comentarios al tema de la simulación: Los accidentes de trabajo y su relación con la medicina legal (BERNAL, 1911); Estudio médico-legal de la incapacidad en los accidentes de trabajo (CALDERÓN REYES, 1929); Medicina Legal (URIBE CUALLA, 1934, 1939); La incapacidad permanente en los accidentes de trabajo (VILLA, 1942); Conceptos que deben reevaluarse para beneficio de la profesión (RESTREPO, 1948); Consideraciones sobre medicina del trabajo e higiene industrial (LLERAS, 1946); La visión en la industria (JIMENO PEÑAREDONDA, 1948); Anotaciones a medicina del trabajo (QUINTERO SANABRIA, 1949); Enfermedades profesionales (CAMARGO, 1954). Asimismo, se analizaron editoriales y artículos de vulgarización publicados principalmente por Pablo Valero Tavera en la revista Salud y Trabajo de la Compañía Colombiana de Seguros de Vida (desde este momento CCSV). Además de las fuentes de otros países, usadas para encuadrar la forma como fue objetivada la cuestión de la simulación en Colombia.

\section{Filiaciones teóricas, definición y clasificación}

Los expertos colombianos en simulación recurren a un grupo más o menos reducido de autores para establecer este campo semántico en la literatura médica. Por un lado, se identificanlos representantes de la psiquiatría francesa y los traumatólogos de la guerra, principalmente Edouard Brissaud, Joseph Babinski, Paul Chavigny, Antoine Porot, Angelo Hesnard ${ }^{6}$. Igualmente se observa la

\footnotetext{
${ }^{5}$ No es del caso entrar a analizar la historiografía. Es suficiente decir que varios de los trabajos mencionados en el texto, así como otros ausentes, analizan o al menos mencionan el debate sobre la simulación en Argentina, Australia, Brasil, España, Francia, Estados Unidos, Inglaterra, Japón, Suiza, entre otros. En el caso de Colombia, no existe ningún antecedente historiográfico sobre el tema de la simulación. Si bien existen algunos historiadores del país que se han preocupado por el tema de la salud de los trabajadores, las temáticas abordadas por ellos distan tanto de la temática nuestra que es innecesario un balance historiográfico.

${ }^{6}$ Referente a la simulación ocasionalmente se mencionan Des maladies simulees et des moyens de les reconnaitre (BOISSEAU, 1870); Psychoses Et Affections Nerveuses (BALLET, 1897); Guide pratique du medecindans les accidents du travail: suites medicales et judiciaires(Forgue, 1909); Traitement des psychonevroses de guerre (ROUSSY e LHERMITTE, 1918); L'Expertise mentale militaire(POROT e HESNARD, 1918). Fuera del círculo de autores franceses otros tuvieron cierta popularidad como Charles Daussat, Miguel Slocker, Donato Boccia y León Lattes. Ninguna de las tesis o artículos analizados referencia publicaciones inglesas, pese a que la simulación (malingering) fue motivó de tratados desde el siglo XIX (WATSON, 1994)
} 
influencia de la psiquiatría alemana, representada por los estudios sobre la simulación y la histeria de Ernst Kretschmer y las neurosis traumáticas de guerra de Hermann Oppenheim, así como los aportes en medicina legal, traumatología y psicopatología del italiano Arnolfo Ciampolini. Finalmente, se percibe la influencia de la psicología española, utilizada en varios casos como fuente indirecta para aproximarse a los teóricos franceses. En efecto, en esta literatura ibérica se destaca la apropiación de ambas escuelas, pero también la observación clínica y la experimentación. Aspectos que en el terreno de los argumentos sirve para enriquecer la discusión con elementos de la psicología aplicada y la práctica médica en el mundo del trabajo. En este grupo sobresalen por un lado Antonio Oller y José Germain, médicos del republicano Instituto de Reeducación de Inválidos del Trabajo ${ }^{7}$. Por otro lado Antonio Vallejo Nájera, el médico castrense principal figura de la medicina franquista ${ }^{8}$.

En el tercer nivel, se destaca un círculo colombiano formado por los médicos, que sin ser grandes teóricos del asunto se habían tornado en las décadas de 1930 y 1940, referentes nacionales de la medicina legal y la medicina del trabajo: Jorge Vergara Delgado, Guillermo Sarmiento López, José Manuel Baena Lavalle, Agustín Arango Sanín, Guillermo Uribe Cualla. Varios de ellos se especializaron en medicina del trabajo en los Estados Unidos, escribieron con regularidad

y en el XX se destacan algunas por el interés sobre la simulación en los casos de accidentes de trabajo (COLLIE e SPICER, 1913). El predominio de la escuela francesa en este tema, concuerda con lo observado por la historiografía colombiana acerca de la historia de la medicina en general (MÁRQUEZ VALDERRAMA, 2005; MIRANDA et al., 1993).

7 Sobre el IRIT existe una amplia bibliografía (HERRERO GONZÁLEZ, 2000; MARTÍNEZ-PÉREZ, 1994, 1998, 2006, 2008; PÉREZ e GALLO, 2006) que muestra sin ambages su importancia para el desarrollo de la medicina del trabajo y la psicología aplicada en España. Fue creado el 4 de marzo de 1922 y comenzó a funcionar en 1924. Tenía como función "mejorar las condiciones de las personas afectadas por una discapacidad" y, además contribuir para "resolver los problemas económicos y de relaciones laborales que las altas tasas de siniestralidad laboral comportaban para los empresarios y el Estado" (MARTÍNEZ-PÉREZ, 2006, p. 354). El modelo, según Martínez-Pérez, estaba marcado por un alto grado de medicalización y por el influjo de la Organización Científica del Trabajo (OCT). En lo médico se promovió la aplicación de los principios de la higiene industrial, la ergonomía, los estudios sobre la fatiga y la prevención de accidentes; igualmente se investigó en traumatología y ortopedia y se idearon prótesis para la rehabilitación de los obreros. En el campo de las ciencias del trabajo, se realizaban test de aptitudes para peritos y obreros; selección, formación y orientación profesional de manera que fuera posible obtener el mejor rendimiento del trabajador.

${ }^{8}$ El franquismo ayudó a Vallejo Nágera a realizar experimentos en campos de reclusión de republicanos, con el fin de rastrear la herencia marxista y perfilar su radical política eugenésica basada en el concepto de hispanidad (CAPUANO e CARLI, 2012; FIOL, PÉREZ, e GUZMÁN, 2008). 
artículos sobre medicina del trabajo y fundaron la Sociedad Colombiana de Medicina del Trabajo en 1946.

Más que una selección arbitraria, estos niveles y círculos de filiación resultan de observar y contrastar sistemáticamente las citas de autoridad. Tal proceso permitió establecer las fuentes que sirvieron de fundamento teórico para los autores colombianos e igualmente para los españoles. El hecho de que fuera reducida a un pequeño grupo de autores la larga historia del concepto de la simulación en la medicina y psiquiatría francesas, muestra el esfuerzo sostenido por resignificar muchos de los conceptos a la luz de los desafíos de la Gran Guerra, el desarrollo de la industria y de la seguridad social. Para Sophie Delaporte toda la terminología médica creada para describir la simulación de accidentes entre los soldados de la Grande Guerra, muestra la voluntad de una rama de la medicina en erigirse como especialidad y afirmar su identidad a través de la apropiación de un lenguaje (DELAPORTE, PROCHASSON, e RASMUSSEN, 2004, p. 225).

Si el concepto de simulación posee contornos indefinidos y aparece como un concepto que es al mismo tiempo problemático y que sirve de referencia para la construcción de un campo de estudios, debemos intentar definir qué es lo que en el periodo estudiado se entiende por simulación y en qué sentido se diferencia del modo como hoy la psiquiatría utiliza ese concepto.

¿Qué és la simulación? De acuerdo con el DSM-IV, entre los problemas que pueden ser objeto de la clínica se definesimulación, como la producción intencionada de síntomas físicos o psicológicos desproporcionados o falsos, motivados por incentivos externos como no realizar el servicio militar, evitar un trabajo, obtener una compensación económica, escapar de una condena criminal u obtener drogas (AMERICAN PSYCHIATRIC ASSOCIATION, 2000, p. 457). En la clasificación actual, la simulación se diferencia de los trastornos facticios, porque en estos últimos la motivación de la enfermedad es la atención médica y el tratamiento. A su vez, se diferencia del síndrome de la histeria, porque la simulación es producida conscientemente. En el caso de los trastornos facticios y los desórdenes de conversión (antes histerias), las motivaciones son internas, en el caso de la simulación son externas (KANAAN e WESSELY, 2010).

La definición del DSM,no es muy diferente dela simulación o "pantomima clínica"9 de los médicos de la primera mitad del siglo XX.

${ }^{9}$ La expresión fue acuñada por Moebius de Leipzig, posteriormente Antonio Vallejo Nágera(1951) se apropia del concepto y lo usa para elaborar su tratado sobre el fenómeno de la simulación de la enfermedad. Publicó sus primeros artículos sobre el tema en los años 1920 y en 1934 aparece la primera edición del libro la enfermedad 
Para ellos la simulación erala acción consciente y voluntaria de fingir, imitar, exagerar o agravar una enfermedad, sus síntomas o la incapacidad resultante con el objetivo preciso de obtener algún tipo de beneficio. La definición contemplaba un principio de utilidad inseparable del acto de simular y, a su vez, una conciencia clara del valor del riesgo y el valor del intercambio.

Hay que insistir, la simulación en la forma pura es un acto consciente y voluntario que tiene siempre una finalidad o un principio de utilidad. ¿Qué pasa cuando el simulador deja de ser consciente y de tener voluntad sobre la simulación? Se vuelve una simulación patológica. Entre los dos extremos existen relaciones íntimas, cualitativas y cuantitativas, "infinitas gradaciones, como matices de un mismo color, dificultando el diagnóstico diferencial entre lo real y lo fingido, entre el fraude clínico y la enfermedad" (VALLEJO NÁGERA, 1951, p. 18).

Em relación con la clasificación de la simulación, no hay unanimidad entre los autores y puede variar según el acento en lo etiológico, la sintomatología, la conducta del simulador y su personalidad. Como la etiología de la simulación es la búsqueda de una indemnización, Antonio Oller y José Germain (1929, p. 336) con Carl Thiemla clasifican en simular un accidente que no se ha hecho en el trabajo; simular una enfermedad que no se ha hecho en el trabajo; achacar un accidente verdadero a una enfermedad antigua; autolesiones; exageración y agravación ${ }^{10}$.

También pensando en lo etiológico, se pueden clasificar los simuladores entre los que deliberada y conscientemente falsifican o inventan un accidente de trabajo o el origen de la lesión; los que fingen una incapacidad o la ocultan o disimulan ${ }^{11} \mathrm{y}$ achacan a un accidente el

simulada. El médico colombiano Jaime Camargo (1954), basa prácticamente todo el capítulo de la simulación en ese libro. No sabemos si para la época el colombiano Camargo Franco conocía de la sombría vida del autor de la simulación.

10 La mayoría de los casos y ejemplos dados por estos médicos, así como por los médicos colombianos, se concentra en el grupo de trabajadores que exageran o que agravan la enfermedad, al pasó que son excepcionales los casos de simulación. Esto se justifica porque simular una enfermedad que no se tiene exige de gran voluntad y perseverancia y pruebas simples como acercar el reóforo al ojo ciego producen en el simulador una reacción instintiva de distanciamiento. Pero también porque el simulador difícilmente puede imitar los síntomas profundos de la enfermedad y reduce la imitación a los más prominentes.

${ }^{11}$ La disimulación es particularmente útil o común en la industria. El obrero sabe que existen gestos, palabras y síntomas que pueden ser obstáculo para conseguir o permanecer en el trabajo, por lo tanto disimula para mostrar que es capaz mental y físicamente de cumplir los requisitos del cargo. Jorge Vergara anota que en el examen de pre-empleo dadas las consecuencias jurídicas y económicas los trabajadores se esfuerzan por ocultar todas las cosas que consideran pueden ser 
deterioro orgánico general; los que exageran los trastornos funcionales con el objetivo de obtener grandes indemnizaciones (JIMENO PEÑAREDONDA, 1948, p. 178-179).

Con respecto a la sintomatología Paul Chavigny describe cuatro especies: a) la simulación en que los síntomas mentidos o alegados son siempre subjetivos, sin manifestaciones externas, el sujeto dice tener "dolor, insensibilidad, impotencia funcional, pérdida de la memoria, desfallecimiento, incapacidad para el trabajo"; b) aquella simulación en que los síntomas consisten en la exhibición de signos objetivos de enfermedad producidos bajo el dominio de la voluntad, por ejemplo tartamudez, temblor, incontinencia urinaria, ataques convulsivos; c) simulación basada en síntomas imitados que prescinden de voluntad mediante el uso de aparatos ortopédicos o productos químicos; d) simulación a partir de síntomas provocados generalmente por médicos y empíricos especialistas en el fraude, en ese caso se aplican inyecciones de parafina o vaselina para simular tumores; sellos de ácido pícrico para simular ictericias; los golpes, frotaciones o quemaduras menores con ácido clorhídrico o fénico; introducción en el tejido subcutáneo de sustancias sépticas para producir flemones; trementina y petróleo para flemones asépticos (CAMARGO, 1954, p. 43; VALLEJONÁGERA, 1951, p. 61).

Del mismo modo, a partir del síntoma, se destaca la clasificación de Gustave Roussy y Jean Lhermitte en simulaciones provocadas, simulaciones creadoras y simulaciones de exageración o perseverancia. Las primeras son provocadas generalmente por agentes químicos o biológicos; las creadoras comprenden actitudes viciosas, trastornos motores y trastornos subjetivos; en las de exageración el sujeto puede prolongar el síntoma después de desaparecida la lesión o ampliar la sintomatología de un trastorno existente(OLLER e GERMAIN, 1929, p. 336; VILLA, 1942, p. 172).

Finalmente, observando la conducta del simulador, Antoine Porot y Angelo Hesnard diferencian simuladores absurdos, pasivos y activos, siendo que los primeros imitan o exageran groseramente una enfermedad psíquica, los segundos adoptan una actitud de mutismo e inabordabilidad, los terceros imitan y producen defectos físicos o temblores y tartamudez (CAMARGO, 1954, p. 42; VALLEJO NÁGERA, 1951, p. 63). Muy cerca de la clasificación psicológica anterior se propone la división entre simuladores ocasionales y perpetuos. Los primeros recurren a la pantomima clínica en ocasiones excepcionales de la vida, se caracterizan por tener un horizonte moral muy 
restringido, un escaso sentido de la propia estimación y del sentido de la dignidad. Los segundos hacen de la pantomima una forma de vida y difícilmente pueden ser clasificados como normales.

El campo semántico de la simulación, aunque complejo y lleno de matices conserva artificiosamente los límites de la sintomatología voluntaria y consciente. Por el contrario, las formas psicopatológicas o las "reacciones psicogéneticas" surgen en el momento en que la simulación se torna involuntaria y subconsciente. Pero la cuestión es menos simple de lo que expresa ese juego de pares dialécticos.

Diversos motivos pueden impulsar a los sujetos a recurrir a la mentira, la ficción y la simulación. El problema es cuando la enfermedad fingida o provocada se vuelve una forma de vida, o cuando el sujeto prolonga la sintomatología de la enfermedad durante años, aún después de obtenida la indemnización o desaparecida completamente la causa productora. De esa manera, el sujeto se aferra tan profundamente a la enfermedad que el factor volitivo es anulado y el simulador termina por "histerizarse" y ser víctima de la superchería. Al final la pantomima ocasional se hace perpetua, un hábito histérico con impacto probable en lo orgánico, las marcas entre lo voluntario e involuntario desaparecen y el proceso psicológico se desvía de su cauce y desarrollo normales.

En esta lógica, principalmente a partir de los trabajos de Joseph Babinski, todas las histerias son especies de simulación psicopatológica $\mathrm{y}$, por tanto, todos los simuladores son susceptibles de ser considerados anormales. Según Vallejo Nágera "las estadísticas demuestran la anormalidad caracterológica de los simuladores [...] y el predominio de los psicópatas, principalmente histeroides" (1951, p. 42). Para él y otros autores, la inferioridad orgánica congénita o la constitución histérica fruto de una herencia neuropática o de estigmas de degeneración,así como la predisposición expresada en una mayor o menor sugestibilidad y autosugestibilidad, desempeñan un papel importante para la eclosión de las formas anormales de simulación. En síntesis, el simulador es anormal puesto que presenta un estado de hipersugestibilidad y una constitución biopsíquica favorable al refugio en la enfermedad.

En la época circularon diferentes clasificaciones o encuadramientos de las formas psicogenéticas de la simulación. Entre las más destacadas estaba la de Ernst Kretschmer. Según este psiquiatra alemán existen cuatro mecanismos psicológicos de simulación: por intención o finalismo, por acostumbramiento histérico, por un refuerzo voluntario de los reflejos o acumulación afectiva y 
sobre una base de disociaciones hipobulicas-hipnoides ${ }^{12}$. En el primer caso, se trata de individuos con ciertas predisposiciones reflejas anormales que les permite voluntariamente vomitar o temblar con enorme facilidad. El acostumbramiento histérico, depende de los procesos fisiológicos automáticos o el aparato ocasional, que permite por ejemplo despertarse a una hora concreta sin necesidad de intervención de la voluntad, el sujeto adopta cierto habito relacionado con una enfermedad el aparato ocasional creado se emancipa y crea una fórmula estereotipada. El refuerzo voluntario de los reflejos consiste en condensar toda la energía, de manera que sea posible aumentar un temblor; en la acumulación afectiva el histérico "busca y utiliza las menores ocasiones de excitación con lo cual consigue alimentar y entretener sus mecanismo reflejos, temblores, palpitaciones, etc.". Por último, las reacciones hipobúlica-hipnoica, son descritas por Kretschmer como la facilidad que tienen ciertos histéricos degenerados o ciertos psicópatas esquizoides de disociar de las capas superiores los mecanismos ps唒uicos profundos, aún con la influencia de excitaciones afectivas ligeras y ocasionales (OLLER E GERMAIN 1929, 328-331; VALLEJO NÁGERA 1951, 35-40).

Si bien la clasificación Kretschmer es citada en prácticamente todas las tesis de medicina del trabajo, entre los médicos legales del país es más probable que se usara la del médico legista Guillermo Uribe Cualla (1934, p. 184-188, 1939, p. 194-199). En primer lugar, porque Uribe Cualla hace parte del grupo de médicos colombianos que legitimaron la medicina legal a través de la docencia y mediante la difusión de los conocimientos teórico-prácticos. En segundo lugar, porque la clasificación es realizada en un manual de medicina legal cuya función es servir de ayuda para las pericias médico-legales. Como afirma Ludwig Fleck, el manual constituye el momento normativo de la ciencia, aquel en que las ideas en disputa se entretejen y coaccionan para establecer una forma definitiva (FLECK, 1986, p. 167-171).

Según Uribe Cualla, las neurosis traumáticas se dividen en histeria post-traumática, la neurastenia traumática, la histeroneurastenia traumática y la sinistrosis, pero aclara que esta última se diferencia de las anteriores, en la medida que el desorden nervioso no es resultado propiamente dicho del traumatismo, sino de la interpretación que el sujeto hace del accidente. Lasinistrosis se ubica en el grupo de las neurosis del deseo o en el cuadro de las histerias

12 En su libro La enfermedad simulada, Vallejo Nágera afirma que estos estados son el resultado de un choque emotivo que se expresa por mecanismos complejos subconscientes e conscientes, estados crepusculares, ensueños, estupor semicatatónico y síntomas de pánico. (VALLEJO NÁGERA, 1951, p. 37). 
traumáticas. Se trata de una forma agravada de la simulaciónexageración, que emerge consecutivamente al deseo de alcanzar una máxima indemnización, eludir una responsabilidad penal o conseguir una determinada finalidad. En la sinistrosis, el sujeto interpreta erróneamente la magnitud del accidente, y considera que el traumatismo tiene graves consecuencias para su desempeño laboral, se presenta el caso incluso de sujetos queterminan por convencerse de que son incapaces para todo tipo de trabajo. La sinistrosis, descrita por Edouard Brissaud a comienzos del siglo XX y vigente en la actualidad, se caracteriza además por una obsesión inimputable al accidente, al riesgo profesional o al dolor. Por tratarse de un estado psicopatológico directamente relacionado con una aspiración precisa, una vez recibida la indemnización desaparecen los síntomas y la herida se cura (CAMARGO, 1954, p. 37-38).

A diferencia de la sinistrosis, las otras neurosis traumáticas se presentan diferidas o aplazadas después del accidente, como efecto del daño psicológico potencial del traumatismo. En los casos de histeria traumática, después del accidente se producen parálisis, crisis convulsivas, contracturas no originadas por el traumatismo, sino por la localización de la histeria. Así por ejemplo, Uribe Cualla cita el caso de un obrero que "diagnosticado erróneamente con una lesión de la medula y una fractura de la pelvis" presentó una "contractura de la cadera en tal grado [...] que andaba muy difícilmente y con la cadera rígida" (URIBE CUALLA, 1939, p. 195). La neurastenia traumática, se caracteriza por el agravamiento inconsciente de los síntomas a medida que el litigio o pleito avanza;el obrero presenta astenia, lagunas, irritabilidad, edemas, cefalea, insomnio, algias localizadas, trastornos dispépticos gastrointestinales, entre otros síntomas. Finalmente, la histero-neurastenia traumática junta los síntomas motores, sensitivos y sensoriales de la histeria con un estado de tristeza, inquietud y preocupación.

Expusimos aquí los diversos modos de entender el concepto de simulación como supuesta línea de demarcación entre lo normal y lo patológico, entre el fraude y la realidad ocultada, sin embargo, se trata, como intentaremos mostrar, de un concepto frágil, que conduce a múltiples equívocos y engaños. Razón por la cual en este escrito asumimos una perspectiva de análisis crítica que permita evidenciar la íntima relación que existe entre esa categoría médica y las estructuras económicas y sociales. 


\section{La construcción social de la simulación}

Hasta ahora se ha mostrado que la forma como la psiquiatría francesa definió y clasificó la simulación tuvo eco en todos los médicos colombianos. En el universo semántico creado en torno a la simulación por los extranjeros y los nacionales, por un lado, se definió el simulador puro con características patológicas más atenuadas en razón de la voluntad y consciencia que determina su acto. En el otro extremo, se clasificaron las formas mórbidas de la simulación, asentadas en la idea de inconsciencia y locura. Ambos extremos hacen parte de una escala peyorativa y de un espectro de condenación en que el sujeto por ser más consciente es más criminal y por ser menos consciente es más loco. Uno de los peligros de la psicologización de la simulación está en la infame proximidad entre la simulación y la histeria, como una línea circular en que sus dos extremos casi se tocan, el salto del crimen a la locura es prácticamente previsible.

Lo paradójico es que al paso que se arraigaba peligrosamente el carácter patológico de la simulación, la confusión y la fragilidad teórica se hacían más evidentes. Al destacar la dificultad del psiquiatra para definir las características y la personalidad de los simuladores, Vallejo Nágera dice que "media gran distancia entre el pueril fraude del estudiante o el obrero que quiere proporcionarse unas vacaciones y la del asesino que se refugia en la locura para salvarse del patíbulo" (1951, p. 43). El comentario apunta aun tema de gran actualidad como es el de la imposibilidad de la psiquiatría para establecer diagnósticos diferenciados. Del mismo modo, insinúa la dificultad de este campo de saber para operar con variables sociales o con problemas,que no pueden ser racionalizados e intervenidos únicamente en términos médicos o psiquiátricos, y que deben integrar nociones de la sociología, la antropología cultural (ROSATTI, 1999), cabe agregar, de la historia.

Aunque la simulación no es exclusiva del mundo del trabajo ${ }^{13}$, si es este un escenario ideal para observar las tensiones frecuentes entre ideología, intereses económicos y ciencia médica. En pocas palabras, es

13 Aunque no es exclusiva la preferencia por los ejemplos con eses grupos sociales marcan la pauta en los teóricos franceses y españoles del siglo XX. Tratados de medicina legal como los del colombiano Guillermo Uribe Cualla(1934, 1939) en los que especies de simulación psicopatológicas aparecen fundamentalmente asociado a los accidentes del trabajo y las enfermedades profesionales, confirman que en la primera mitad del siglo XX se trató de un problema profundamente vinculado al mundo laboral. Esto concuerda con la visión de Antonio Oller y José Germain para quienes la simulación como problema médico y social coincide con el nacimiento del servicio militar obligatorio y la promulgación de las leyes de accidentes (OLLER e GERMAIN, 1929). 
un escenario ideal para comprender la construcción social de la enfermedad.

En primer lugar se debe recordar el principio de utilidad que se atribuye a la simulación o pantomima clínica. De tal principio se desprende un presupuesto que es que el simulador siempre tiene por objetivos incrementar el importe de la indemnización, librarse del servicio militar, ganar un subsidio de enfermedad, obtener una póliza de seguro, ganar unas vacaciones, conseguir más medicamentos de los necesarios. En ese punto la pretensión científica se rompe para exhibir con claridad el áspero sustrato ideológico.

En efecto, a la vista de los médicos de la época la simulación es ante todo un defecto de la virtuden la clase obrera, en los pobres, los soldados y los inmigrantes. Al final lo que hace la clasificación psiquiátrica es reforzar con retórica científica la ideología de una época. A los pobres se les atribuyó el uso de la enfermedad simulada como medio ilícito de obtener de la caridad, la beneficencia pública y la filantropía los medios para su sustento.

A los soldados se imputó la práctica desde comienzos del siglo XIX y durante la Gran Guerra; aun cuando los médicos del Centro Médico Legal de Amiens por prudencia o indulgencia apenas condenaron por autolesión al $5 \%$ de los $\operatorname{soldados}^{14} \mathrm{y}$, en el frente contrario, médicos como Hermann Oppenheim arguyan que las neurosis traumáticas de guerra, eran un deseo inconsciente yno un fraude (WESSELY, 2003, p. 38).

En los inmigrantes la simulación es prácticamente endémica. A mediados del siglo XIX se consideraba que los germano-americanos eran una población particularmente afectada por la "simulación", pero dos décadas antes era un problema de los irlandeses y posteriormente fue de los polacos y los judíos (KANAAN e WESSELY, 2010). En el siglo $\mathrm{XX}$, los inmigrantes del norte de África fueron vistos como sospechosos de aprovecharse de la generosidad del Estado francés mediante la simulación de enfermedades o accidentes (FERREIRA, 2007).

Sin embargo, la clase obreraparece haber sido más sensible a los "efectos corrosivos" del estado de bienestar y en consecuencia más dispuesta a la simulación en el siglo XX. Esa parece ser al menos la impresión de los contemporáneos, para quienes los seguros de enfermedad y de accidentes suscitaron en los trabajadores un ansia

14 Según Sophia Delaporte (2004) entre 1914-1918, el Centro Medico Legal de Amiens condenó el 5\% de los soldados por autolesión, en el 25\% de los casos la pericia no fue concluyente, el $65 \%$ creyó que no había razones para pensar que se trataba de una forma de simulación o autolesión para evitar el frente de batalla. 
ilegitima de beneficios econômicos (COLLIE e SPICER, 1913; OLLER e GERMAIN, 1929; WESSELY, 2003).

Especie de epidemia, la simulación atemorizo a médicos y conservadores alemanes, ingleses, franceses y españoles, para quienes el alarmante incremento de los accidentes de trabajo ${ }^{15}$ era consecuencia de una legislación que desfavorecía las clásicas virtudes y fomentaba las reivindicaciones de la clase obrera. Arnolfo Ciampolini, conocido y citado en varias de las obras y artículos alusivos a la simulación, afirmaba al respecto que el trabajador simulador eraun holgazán, un sujeto que eludía sus compromisos sociales y profesionales (VALLEJO NÁGERA, 1951, p. 41).

En conclusión, como dicen Kanaan y Wesseley, en la historia de la histeria y la simulación se revela una notable dicotomía: "mientras que distinguir las condiciones por medios clínicos o psicológicos puede ser muy difícil, puede ser muy fácil distinguir las condiciones de género o de clase" (2010, p. 72). Afirmación que recuerda la moral biológica que inspiró la medicalización de las denominadas enfermedades sociales (alcoholismo, tuberculosis y sífilis), durante las primeras décadas del siglo XX. En ellas así como en la simulación, la incertidumbre científica es tan grande como la condena social y los preconceptos que promueven.

Los médicos colombianos comparten con sus homólogos extranjeros, la misma preocupación por el aumento de la accidentalidad laboral a causa de la simulación. De hecho, las ideas preconcebidas acerca del uso ilegítimo de los derechos sociales ya están presentes desde las primeras publicaciones sobre el tema, incluso se anticipan a la formulación de la ley de accidentes. Así, en 1911, Benjamín Bernal se preguntaba "qué no será entre nosotros", sí un obrero francés con fractura de antebrazo tarda doscientos días en recuperarse cuando está asegurado y por término medio diecinueve cuando no tiene seguro (BERNAL, 1911, p. 35). Para Bernal, el principal problema es que el obrero le toma gusto al ocio. Por lo tanto, el médico en el "importante papel social que desempeña" no debe ser indiferente al descanso abusivo y debe "salvaguardar los intereses financieros de la empresa" y evitar "al obrero las consecuencias de su inactividad" (BERNAL, 1911, p. 38).

En las décadas siguientes, comentarios parecidos a los de Bernal aparecen en revistas académicas y de vulgarización, así como en

\footnotetext{
15 Según Wesseley (2003, p. 33) la cuestión que debe llamar la atención no es el aumento de los casos sino el hecho de que cuando los médicos hacen el comentario olvidan que la relación entre patrones y obreros era desfavorable para estos últimos y las leyes de compensación era la única forma de equilibrar la situación.
} 
las tesis elaboradas por los médicos de la Universidad Nacional de Colombia y la Universidad de Antioquia. Estos documentos repiten las observaciones y comentarios médicos extranjeros y destacan la gravedad del problema sin mayores justificaciones económicas o datos empíricos.

Pablo Valero Tavera no duda en señalar que la simulación es un grave problema social. Esto lo reitera de diferentes formas en la revista Salud y Trabajo de la CCSV o en la Revista de Medicina Legal de Colombia órgano del Instituto de Medicina Legal de Bogotá. Para Valero (1948), está demostrado hasta la saciedad que muchos obreros fingen dolores, limitaciones de movimiento o provocan accidentes con el "fin exclusivo de obtener el pago de los salarios por incapacidad". Pese a la contundencia de la afirmación, las opiniones parecen estar divididas. Roberto Lleras (1946, p. 65) considera a los obreros colombianos incapaces de sacrificar un brazo o una pierna para obtener uno o dos años de salario. De la misma opinión es Osvaldo Avello (1948, p. 29), que nunca presenció casos en sus labores de inspector del trabajo en Pasto, Villavicencio y Barrancabermeja. Pero el médico Gonzalo Arango Barreneche (1957, p. 52), afirma que no es exótico que los asalariados se mutilen voluntariamente para sacar provecho y pone como caso los mineros de la Frontino and Bolivia Gold Mines que se amputaron un dedo para cobrar la indemnización.

Valero soporta su afirmación en las estadísticas recogidas por la CSSV entre 1943 y 1947. De acuerdo con las cifras en un periodo de 5 años se había quintuplicado el número de accidentes y el "índice de frecuencia de la accidentalidad" había pasado de 26.7 en 1943 a 37.6 en 1947 (VALERO TAVERA, 1949a) ${ }^{16}$.

\begin{tabular}{c|r|r|r}
\hline & $\begin{array}{c}\text { Número de } \\
\text { accidentes }\end{array}$ & $\begin{array}{c}\text { Número de } \\
\text { Asegurados }\end{array}$ & $\begin{array}{c}\text { Índice de } \\
\text { Frecuencia de } \\
\text { la } \\
\text { accidentalidad } \\
\text { c/100 } \\
\text { trabajadores }\end{array}$ \\
\hline 1943 & 1883 & 7057 & 26.7 \\
\hline 1944 & 2182 & 8433 & 25.9 \\
\hline 1945 & 3214 & 13661 & 23.5 \\
\hline 1946 & 5352 & 21048 & 25.4 \\
\hline 1947 & 9790 & 26040 & 37.6 \\
\hline
\end{tabular}

${ }^{16}$ El mismo artículo fue reproducido al año siguiente en la Revista de Medicina Legal de Colombia (VALERO TAVERA, 1949b). 
El fenómeno se explica según Valero por la promulgación de la Ley 6 de 1945 (decretos reglamentarios 841 y 1288 de 1946) que fijó las indemnizaciones por concepto de Incapacidades Permanentes producidas por Accidentes de Trabajo. Desde su punto de vista o en el de otros médicos (CAMARGO, 1954, p. 39; JIMENO PEÑAREDONDA, 1948, p. 177; QUINTERO SANABRIA, 1949, p. 72), los incentivos sociales habían servido de acicate para la simulación al elevar dos terceras partes el pago del salario completo durante el periodo de incapacidad. "Pues si bien es cierto que al trabajador enfermo no se le disminuyen sus obligaciones, es un hecho que existe la tendencia al ocio y es agradable recibir una remuneración sin que haya sido ganada con el sudor de la frente"(VALERO TAVERA, 1948).

La ausencia de estadísticas oficiales sobre cualquier cuestión laboral, no permite objetar los comentarios de Valero acerca de um supuesto influjo de la simulación en el número de accidentes y, por ende, en el "índice de Frecuencia de la accidentalidad c/100 trabajadores". En todo caso, hay que decir que Valero confunde la "frecuencia" con la "incidencia" o la tasa de accidentalidad. Para evaluar la frecuencia de la accidentalidad, él debería haber considerado el número de accidentes por cada millón de horas trabajadas, un cálculo que ya era común realizar en la época (ARAGÓN e ARCHILA, 1934). De hecho las cifras de Valero son poco útiles para representar la dimensión del problema. Lo único que se puede concluir de ellas, es que en 1946 prácticamente se duplicó el número de asegurados y que ese año y el siguiente salieron de la "inseguridad social" 12.379 obreros. En consecuencia, al mayor número de asegurados corresponde un mayor número de accidentes, que pueden salir del anonimato o del sub-registro para comenzar a engrosar las rudimentarias estadísticas.

Hay que decir también respecto de estas cifras, que el recurrente uso de modelos estadísticos aquí señalado, independientemente de los problemas metodológicos destacados, padece de una dificultad que no se limita al simple uso inadecuado de conceptos tales como los de frecuencia o incidencia. La dificultad mayor de estos estudios es que ellos pretenden establecer mediciones en torno a un concepto ambiguo e imposible de ser delimitado como es el concepto de "simulación". Aquello que Desrosiéres (2008) denomina una "convención colectiva de equivalencia", no llega a ser definida como tal en el caso de la simulación como ocurriría con categorías previamente definidas, tales como el índice de pobreza. Sin embargo el concepto de simulación, si bien continúa siendo controvertido y ambiguo, pudo servir de base para la decisión de negar auxilios 
sociales. Aun cuando se tratase de observaciones poco consistentes y de estudios estadísticos poco confiables.

Más allá del problema del uso retórico de la cifra en Colombia ${ }^{17}$, es necesario destacar algunos elementos sobre los estudios relacionados con la salud de los trabajadores en Colombia, durante las décadas de 1930 y 1940. Así se puede localizar y valorar el lugar de Valero en el contexto de nacimiento de una práctica discursiva como la medicina del trabajo ${ }^{18}$. Si pensamos en términos de producción sistemática de conocimiento, se puede decir que era muy incipiente la investigación colombiana del periodo. De acuerdo con el número de publicaciones (artículos y tesis) se trató principalmente de monografías o reflexiones teóricas sobre seguridad social (23), accidentes de trabajo (21), medicina del trabajo (16), enfermedades profesionales (12), silicosis (11), ferrocarriles (7), bananeras (6), higiene industrial (3), petroleras (3), barrios operarios (2) yhaciendas cafeteras (2).

Valero así como Jorge Vergara, José Miguel Baena Lavalle, Guillermo Sarmiento, Agustín Arango Sanín, entre otros, formaban parte del reducido círculo de especialistas en cuestiones laborales, y por lo tanto, de un círculo de sociabilidad siempre en contacto directo con el Departamento Nacional del Trabajo. Así por ejemplo, en 1947, Baena y Sarmiento, fungían de director y subdirector de la Sección Nacional de Medicina e Higiene Industrial del Ministerio del Trabajo. De modo que los debates legislativos y técnicos sobre enfermedades profesionales como la tuberculosis, la hernia o la anquilostomiasis pasaban necesariamente por su aprobación.

A su vez, Sanín, Valero y Vergara formaban parte del comité editorial (director, subdirector y redactor respectivamente) de la revista Salud y Trabajo de la Compañía Colombiana de Seguros de Vida. La revista, había sido creada en 1947 con el objetivo de servir de consulta y de orientación sobre las prestaciones sociales. Basados en la experiencia y el estudio "científico" de los problemas, sus redactores pretendían convertir la revista en el principal medio de vulgarización de cuestiones como legislación laboral, accidentes de trabajo, medicina del trabajo, higiene industrial, prevención, estadística laboral. En otras palabras, la revista Salud y Trabajo, a falta de órganos académicos especializados en medicina del trabajo, abanderaba la discusión nacional sobre salud y trabajo. Por sus páginas pasaron todos los

${ }^{17}$ Sobre esta especie de uso retórico del dato estadístico véase: (ESTRADA, GALLO, e MÁRQUEZ, 2015; MARTÍNEZ e GUATIBONZA, 2005).

18 Más detalles acerca del proceso de configuración de la medicina del trabajo en Colombia (GALLO, 2014). 
citados autores, además de otros miembros y médicos fundadores de la Sociedad Colombiana de Medicina del Trabajo, creada en 1946.

Cabe destacar que las revista académicas, es decir las vinculadas a las facultades de medicina o ingeniería, publicaron sobre el tema eventualmente. Así pues, quien quería estar al tanto de la discusión debía leer Salud y Trabajo, El Boletín del Departamento Nacional del Trabajo, creado en 1927, y la Revista de Medicina Legal.

Por otro lado, no es difícil imaginar que la promulgación de la ley generó cierto revuelo entre los industriales y los trabajadores. Que el número de afiliados se haya casi cuadriplicado se podría justificar en el crecimiento de laindustria colombiana, pero es obvio que la ley debió presionar algunos sectores a menudo reticentes a cumplir las leyes laborales. En cuanto a los trabajadores, la ley 6 de 1946 que unificó la legislación laboral incluyendo todo lo relativo a prestaciones sociales y de salud, debió generar una especie de impulso reivindicatorio en el sentido de empoderamiento para la reclamación de los derechos.

Finalmente, es interesante destacar que el aumento de los accidentes de trabajo también puede ser resultado de cambios inherentes al modelo de producción o la organización del trabajo. Desde 1925, la empresa de cervezas Bavaria y otras industrias colombianas con capitales importantes, instauraron seguros de vida colectivos para empleados y trabajadores (LÓPEZ-URIBE, 2011, p. 184). La CCSV, a la cual hacen referencia las estadísticas de Tavero, era la aseguradora de esta industria cervecera. En esta industria, se presentaron cerca de 2000 accidentes de trabajo en 1945. Según Alberto Mayor Mora, la accidentalidad parecía estar asociada con la salida de los técnicos alemanes, pero la creación en 1946 de la Oficina de Seguridad y la instauración de un intenso programa de control y de estímulos "morales y materiales", permitió que la accidentalidad se redujera a 789 en 1946 y 301 en 1947(MAYOR MORA, 1997, p. 174175). Hay muchas inconsistencias en esos datos y por lo mismo se puede dudar de la eficacia de la estrategia industrial para disminuir la accidentalidad ${ }^{19}$. Pero todo eso es irrelevante en este momento, lo que hay que resaltar es que la accidentalidad puede crecer o decrecer en razón del modelo productivoy no por la astucia de unos cuantos simuladores.

19 Por el texto de Mayor Mora no se sabe cuántos empleados tenía la empresa cuando ocurrieron los 2000 accidentes. Es de suponer, que esa cifra corresponde a toda la empresa y sus consorcios dispersos en varias regiones del país. Esto porque la sede principal en Bogotá tenía 1280 obreros en 1945. De todas formas una accidentalidad de esas es alarmante. Para tener una idea, en 1928, la Tropical Oil Company registró 1136 accidentes en 4000 trabajadores. 
Lo dicho acerca de los trabajos de Valero corrobora la idea de una construcción social del problema de la simulación. Si en la psiquiatría la clasificación funciona como un dispositivo para reforzar la anormalidad y peligrosidad de los sujetos. En el contexto de los seguros de vida el obrero simulador pasa a ser hijo de la astucia y no de la patologia (VILLA, 1942, p. 172) ${ }^{20}$. De la cifra se salta así a lo ideológico y político. Como dice Victoria Haidar refiriéndose a la forma como los médicos argentinos objetivaron el problema de la simulación, mientras para la moral burguesa representada por los médicos"la salud revestía un carácter sagrado, los trabajadores podían llegar a 'vender' una parte de sus cuerpos"(HAIDAR, 2013, p. 101). Pero los obreros no se pueden cargar con todo el peso de la culpa por profanar su cuerpo, ellos suelen ser "seres ignorantes que no han oído la voz amiga que los haga caer en cuenta del grave error que incurren con tal proceder" (VALERO TAVERA, 1948). Ese fue precisamente el grave error que cometió el legislador colombiano, "no haber instruido a los obreros previamente"(QUINTERO SANABRIA, 1949, p. 72).

Al final solo resta tratar de elevar el nivel cultural del obrero. Combatir la idiosincrasia de un pueblo. Evitar que el obrero ingrese en las filas de los mendigos o los maleantes. Pero sobre todo evitar que se vuelva una carga o un estorbo social y que contribuya con su trabajo al bienestar colectivo y la prosperidad de la patria. Esto se puede hacer desde la escuela, en la fábrica o el sindicato. El mensaje debe ser claro, visible y persuasivo, como los que exhibe la revista Salud y Trabajo en cada una de sus páginas: "La indemnización es siempre inferior a la más insignificante parte de su cuerpo", "Los simuladores adquieren remedios, pero se perjudican cuando están enfermos", "La simulación de accidentes a la larga solo termina perjudicando al mismo trabajador", "El dinero de la indemnización se acaba pronto, la incapacidad lo acompaña de por vida".

\section{Conclusiones}

En la primera parte de este artículo se analizaron las filiaciones, la definición y clasificación de la simulación. Para ello se usaron

${ }^{20}$ Vale la pena destacar la diferencia que existe en relación al tratamiento de otras formas de simulación, tal como ocurrió con la simulación de la locura. Sobre esta cuestión existe una amplia literatura que se inicia a finales del siglo XIX, tanto en Europa como en América Latina. En Europa podemos destacar autores como Magnan(1887) o Laurent (1866) y en América Latina podemos mencionar los textos del psiquiatra argentino José Ingenieros (1903) La Simulación de la Locura, y del médico colombiano Guillermo Uribe-Cualla (1939) Medicina Legal y Psiquiatría Forense, ambos interesados en el problema de la simulación de la locura y la responsabilidad criminal. 
principalmente fuentes colombianas y algunas europeas, teniendo en este último caso cuidado de usar exclusivamente aquellos autores que eran referenciados o citados por los colombianos. De esta manera intentamos aproximarnos a la forma como los médicos pensaron y objetivaron el problema de la simulación desde la academia. Ahora, en la segunda parte se observó cómo ese conocimiento académico fue atravesado por intereses económicos y factores ideológicos. Concretamente se pudo constatar que el conocimiento producido en torno al tema por parte de médicos vinculados a las empresas de seguros, determinó la manera en que circuló y se apropió la simulación en Colombia ${ }^{21}$.

Pero más que resumir algunas de las cuestiones ya anotadas, cabe plantear varias reflexiones. En primer lugar se percibe en la forma en que fue resuelta la cuestión de la simulación un llamativo desplazamiento de lo fisiológico a lo psicofisiológico. Tal movimiento obedece a una paulatina "psicologización" de la medicina del trabajo, desplazamiento que se insinúa en el auge de la orientación profesional y la psicotecnia y en la perdida de vigencia de la metáfora del motor humano en función de la del factor humano. Si bien esto ha sido investigado para otros países, hasta ahora la historiografía colombiana no se ha preguntado por los saberes que incursionaron en el mundo del trabajo durante la primera mitad del siglo XX. Entre ellos la psicotecnia.

En segundo lugar, se observa que la simulación en el contexto de la seguridad social funciona como un dispositivo de regulación de servicios o privación de derechos. Esta perspectiva responde con mayor precisión a las necesidades de encuadramiento cualitativo de los usuarios de la seguridad social y los intereses económicos que rigen el funcionamiento de las compañías de seguros.

En tercer lugar, la simulación parece hacer parte de un conjunto más amplio de respuestas o reacciones del trabajador al ambiente opresivo generado por los modelos racionales de producción. En ese conjunto caben otras prácticas como el ausentismo, la parsimonia, el aumento de los tiempos muertos, las manifestaciones de disgusto, la rotación laboral, el consumo de alcohol y de sustancias psicoactivas. Se puede entonces decir como hipótesis para futuras reflexiones, que la simulación es un acto de resistencia o de independencia de espíritu frente a las formas de explotación capitalista, como por ejemplo el trabajo a destajo.

\footnotetext{
${ }^{21}$ Sobre la idea de encuadramiento cualitativo de los usuarios del sistema de salud, véase(FERREIRA, 2007). La autora discute como variables sociales o raciales pueden determinar el acceso a la salud o el reconocimiento de los riesgos profesionales.
} 
Al terminar queda la impresión de que no se respondió completamente a varias preguntas. La más obvia, ¿hubo o no casos de simulación en Colombia durante este periodo? Seguramente, pero exceptuando la mención a las autolesiones de los mineros el resto de las afirmaciones de los médicos al respecto no pasan de especulaciones tendenciosas. ¿Es posible calcular el costo económico de la simulación? No porque cada accidente es un universo de posibilidades y la invalidez profesional puede cambiar de un sujeto a otro. Sin embargo, en la industria cuestiones como el rendimiento, la productividad y la competitividad son muy valorizadas por lo que cualquier accidente representa una ausencia y un obstáculo en la cadena productiva. Por último, ¿por qué estudiar la simulación de la manera como se hizo en este artículo? Porque permite comprender la manera como se crean "falsos" problemas sociales o apropian ideologías científicas para reivindicar una forma concreta de intervención social. También porque al analizar el proceso de formación y apropiación de la simulación en Colombia se observa la "condicionalidad histórica" de una supuesta elección empírico-teórica. Fundamentalmente porque ante la necesidad de evitar un fraude se construyeron discursos, saberes y estrategias de control de los trabajadores que se articularon en torno al eje de la simulación. Una serie de discursos sobre lo normal y lo patológico, con pretensiones de cientificidad, que ponen en evidencia complejas redes de saber y poder. Pero también, porque analizar la simulación en Colombia, en tanto que problema psicológico y desafío del mundo del trabajo, es una forma de aproximarse al proceso de configuración de un saber preocupado por los cuerpos en riesgo ${ }^{22}$.

\section{Referencias}

ABELLO NOGUERA, O. Accidentes del trabajo, vacios de la legislación y la seguridad social. Tesis para optar al título de doctor en Derecho. Universidad Javeriana. Bogotá, 1948.

American Psychiatric Association.Diagnostic and Statistical Manual of Mental Disorders: DSM-IV-TR. 4th ed. Washington, DC: American Psychiatric Association, 2000.

ARAGÓN, V. e ARCHILA, M. Informe Que Rinde El Interventor Nacional de Petróleos y El Jefe de La Oficina General Del Trabajo Al Señor Ministro de Industrias Sobre Visita Practicada en la Instalaciones Petrolíferas de Barrancabermeja. Boletín de La Oficina Nacional Del Trabajo, V (39-44): 69-90, 1934.

22 Véase (CANGUILHEM, 2005; FOUCAULT, 1979). 
ARANGO BARRENECHE, G. Estudio sobre medicina industrial en Medellín. Tesis Doctorado en Medicina y Cirugía. Universidad de Antioquia. Medellín, 1957.

BALLET, G. Psychoses et affections nerveuses; leçons de clinique médicale.Paris: Doin, 1897.

BERNAL, B. Los Accidentes de Trabajo en sus relaciones con la medicina legal. Tesis para optar al título de doctor en Medicina y Ciencias Naturales. Universidad Nacional de Colombia, Bogotá: Casa editorial Arboleda e Valencia, 1911.

BOISSEAU, E. Des maladies simulees et des moyens de les reconnaitre lecons professees au Val-de-grace. Paris: J.-B. Bailliere, 1870.

BRUNO, A. A Perícia Médica nos casos de simulação em Infortunística (Tese Apresentada Ao Primeiro Congresso Brasileiro de Direito Social em 13-V1941, Sustentada Perante a D. Subcomissão de Acidentes do Trabalho e debatida em 17-V-1941). Revista Medicina, June, 9-22, 1941.

CALDERÓN REYES, J. Estudio Médico-Legal de la Incapacidad en los accidentes de Trabajo. Tesis Medicina. Universidad Nacional de Colombia. Bogotá, 1929.

CAMARGO, J. Enfermedades Profesionales. Tesis doctor en medicina. Universidad Nacional de Colombia. Bogotá, 1954.

CANGUILHEM, G. Ideología y racionalidad en la historia de las ciencias de la vida: nuevos estudios de historia y de filosofía de las ciencias. Buenos Aires: Amorrortu, 2005.

CAPUANO, C., e CARLI, A. Antonio Vallejo Nágera (1889-1960) y la Eugenesia en la España Franquista: cuando la ciencia fue el argumento para la apropiación de la descendencia. Revista de Bioética yDerecho, n. 26: 3-12, 2012.

COLLIE, J., e Spicer, A. Malingering and Feigned Sickness.London: E. Arnold. http://archive.org/details/malingeringfeign00colliala, 1913.

DEL VALLE, P. La Medicalización de la justicia en Antioquia (1887-1914). Medellín: Editorial Universidad de Antioquia, 2010.

DELAPORTE, S., Prochasson, C. e Rasmussen, A. Discours médical et simulation. In Vrait et fauxdans la Grande Guerre. Espace de l'histoire. Paris: La Découverte, 2004.

DESROSIÈRES, A. Pour une sociologie historique de la quantification: l'Argument statistique I. Paris: Presses de l'Ecole des mines, 2008. 
ESTRADA, V., GALLO, Ó., MÁRQUEZ, J. Tuberculosis, estadística y mundo laboral en Colombia, décadas de 1930 y 1940. História, Ciências, Saúde Manguinhos (aguardando publicación), 2015.

FERLA, L. Feios, Sujos E Malvados Sob Medida. A Utopia Médica Do Biodeterminismo, São Paulo (1920-1945). São Paulo: Alameda, 2009.

FERREIRA, C. Retour Sur La Sinistrose, Dite Névrose de Revendication. Carnets de Bord En SciencesHumaines, vol. 13: 78-87, 2007.

FIOL, E., FERRER PÉREZ, V., e NAVARRO GUZMÁN, C. La Psicología de Las Mujeres Republicanas según el Dr. Antonio Vallejo Nájera. Revista de Historia de La Psicología, vol.29, n. 3: 35-40, 2008.

FLECK, L. La génesis y el desarrollo de un hecho científico: introducción a la teoría del estilo de pensamiento y del colectivo de pensamiento. España: Alianza Editorial, 1986.

FORGUE, É. EJEANBRAU, É. Guide pratique du médecin dans les accidents du travail, leurs suites médicales et judiciaires. Paris: Masson, 1909.

FOUCAUlT, M. El Poder Psiquiátrico. Curso en el Collège de France (1973-1974). Buenos Aires: Fondo de Cultura Económica, 2007.

GALLO, Ó. Higiene industrial y medicina del trabajo en Colombia, 1912-1948. In: H. CARDONA RODAS, H. ePEDRAZA GÓMEZ, Z. (org). Al otro lado del cuerpo. Estudios biopolíticos en América Latina. Bogotá: Universidad de los Andes, 2014.

GALLO, O. Trabalho, medicina e legislação na Colômbia, 1910-19146. Tese. (Doutorado em História). Universidade Federal de Santa Catarina, 2015.

HAIDAR, V. Las Pérdidas de Jornadas de Trabajo por enfermedad, unapreocupación de larga duración (Argentina, 1930-2012). Gaceta Laboral (Maracaibo), vol. 19, n. 1: 78-124, 2013.

HERRERO GONZÁLEZ, F. Mercedes Rodrigo: una pionera de la psicología aplicada en España y en Colombia. Tesis. (Doctorado en Filosofía) Madrid: Universidad Complutense de Madrid. 2000.

INGENIEROS, J. Simulación de la locura. In: Obras completas, Octava, I. Buenos Aires: Mar Oceáno, 1903.

JIMENO PEÑAREDONDA, A. La visión en la industria. Bogotá: Universidad Nacional de Colombia, 1948. 
KANAAN, R., e Wessely, S. The Origins of Factitious Disorder.History of the Human Sciences, vol.23, n. 2: 68-85, 2010.

LAURENT, A. Étude Médico-Légale Sur La Simulation de La Folie. Considérations Cliniques et Pratiques a L'usage es Médecins Experts, es Magistrats et Jurisconsultes. Paris: Victor Masson et fils, 1866.

LLERAS, R. Consideraciones sobre medicina del trabajo e higiene industrial. Bogotá: Universidad Nacional de Colombia, 1946.

LÓPEZ-URIBE, M. Salarios, vida cotidiana y condiciones de vida en Bogotá durante la primera mitad del siglo XX. Bogotá: Universidad de los Andes, Facultad de Ciencias Sociales-CESO, Departamento de Historia, 2011.

MAGNAN, V.Leçons cliniques sur le maladies mentales. Paris: Baillére, 1887.

MÁRQUEZ VALDERRAMA, J. Ciudad, Miasmas y Microbios. La irrupción de la ciencia pasteriana en Antioquia. Medellín: Universidad de Antioquia. 2005.

MARTÍNEZ-PÉREZ, J. La Organización Científica del Trabajo y las estrategias médicas de seguridad laboral en España (1922-1936). Dynamis. Acta Hispanica Ad Medicinae Scientiarumque Historiam Illustrandam, n. 14: 131-58, 1994.

MARTÍNEZ-PÉREZ, J. Restableciendo la Salud del Estado: Medicina y Regeneración Nacional en torno a un proceso judicial en la encrucijada de los siglos XIX al XX.Dynamis. Acta Hispanica Ad Medicinae Scientiarumque Historiam Illustrandam, n. 18: 127-56, 1998.

MARTÍNEZ-PÉREZ, J. El Obrero Recuperado: Medicina del Trabajo, Ortopedia y Tecnología Médica en la imagen social de las personas con discapacidades (España, 1922-36).História, Ciências, Saúde-Manguinhos, vol. 13, n. 2: 349-73, 2006.

MARTÍNEZ-PÉREZ, J. El 'Factor Humano' y la distribución sexual del trabajo en el discurso de la Organización Científica del Trabajo (España, 1922-1926). Asclepio. Revista de Historia de la Medicina y de la Ciencia, vol., LX, n. 1: 103-28, 2008.

MARTÍNEZ-PÉREZ, J., e PORRAS GALLO, M. Hacia una nueva percepción social de las personas con discapacidades: Legislación, Medicina y Los Inválidos del Trabajo en España (1900-1936). Dynamis: Acta Hispanica Ad Medicinae Scientiarumque Historiam Illustrandam, n. 26: 195-219, 2006.

MAYOR MORA, A. Ética, Trabajo y Productividad en Antioquia. Bogotá: Tercer Mundo Editores, 1997. 
MIRANDA, N., QUEVEDO, E., HERnÁNDEZ, M. et al. Historia Social de la Ciencia en Colombia. Tomo VIII, 2. Medicina. La Institucionalización de la Medicina en Colombia. Santafé de Bogotá: Colciencias. Instituto Colombiano para el Desarrollo de la Ciencia y la Tecnolog苗 Francisco José de Caldas, 1993.

MORALES, E. Algunas Consideraciones Sobre La Fisiología Industrial, La Fatiga Y Accidentes de Trabajo. Tesis para optar al título de doctor en medicina y cirugía, Universidad Nacional de Colombia, Bogotá, 1937.

OLLER, A., e GERMAIN, J. La simulación en general y desde el punto de vista neurológico. In: Oller, A. La prácticamédica en los accidentes del trabajo. Madrid: Morata, 1929.

PASTORE, A. MaladiesVraies et MaladiesSimulées. Les Opinions des Juristes et des Médecins (XVIe-XVIIe Siècles). Equinoxe, n. 22: 11-26, 1999.

POROT, A., e HESNARD, A. L'expertise mentale militaire. Paris: Masson, 1918.

QUINTERO SANABRIA, T. Anotaciones a Medicina del Trabajo. Bogotá: Universidad Nacional de Colombia, 1949.

RESTREPO, JM. Conceptos que deben revaluarse para beneficio de la profesión. Anales de la Academia de Medicina de MedellínII (Tercera Época), n. 6: 486-97, 1948.

ROSATTI, P. La douleur, aux confins du médical, juridique et sociologique. Douleur et Analgésie, vol., 12, n. 1: 1-2, 1999.

ROUSSY, G., e LHERMITTE, J. Traitement des psychonévroses de guerre. Paris: Masson, 1918.

TOUS LIÑÁN, E. Interés Medico-Legal de los Accidentes de Trabajo. Clínica Vargas, vol., 1, n. 6-8: 44-46, 1953.

URIBE CUALLA, G. Medicina Legal. Bogotá: Editorial Nueva, 1934.

URIBE CUALLA, G. Medicina Legal y Psiquiatría Forense. Bogotá: Libreria Voluntad, 1939.

VALERO TAVERA, P. Un Problema Social.Salud y Trabajo, vol. I, n. 4: 1, 1948.

VALERO TAVERA, P. Observaciones a la tabla de valuación de incapacidades y al Régimen de Accidentes del Trabajo.Salud y Trabajo, vol. I, n. 7: 3-8, 1949a.

VALERO TAVERA, P. Observaciones a la tabla de valuación de incapacidades y al Régimen de Accidentes del Trabajo. Revista de Medicina Legal de 
Colombia. Órgano de La Oficina Central de Medicina Legal de Bogotá, vol. XI, n. 57-58: 168-85, 1949b.

VALLEJO NÁGERA, A. La enfermedad simulada. 1a Edición de 1934. Barcelona: Salvat, 1951.

VILLA, G. La incapacidad permanente en los accidentes de trabajo. Tesis Doctorado en Medicina y Cirugía, Universidad Nacional de Colombia, Bogotá, 1942.

WATSON, S. Malingerers, the 'Weakminded' Criminal and the 'Moral Imbecile': How the English Prison Medical Officer Became an Expert in Mental Deficiency, 1880-1930. In: CLARK, M., e CRAWFORD, C.Legal Medicine in History, Cambridge; New York: Cambridge University Press, 1994.

WESSELY, S. Malingering: Historical Perspectives. In: Halligan, P., Bass, C., e Oakley, D. Malingering and Illness Deception. New York: Oxford University Press, 2003.

Recebido em 19 de agosto de 2015

Aprovado em 20 de dezembro de 2015

\title{
The simulation in the world of work, Colombia 1911- 1957
}

\begin{abstract}
The 57 Colombian Law of 1915 stipulated that medical doctors should be responsible for evaluate the magnitude of work accidents. Besides determining the time of disability and the indemnification, they had the function of revealing, in entrance examination, the constitutions or morbid predispositions to the accident of each worker; and they also had to detect cases of "simulation" after the accident. In this article, we analyze how occupational physicians of Colombia tried that simulation. We show that the way in which French psychiatry defined and classified the simulation influenced the majority of Colombian doctors; although in legal medical practice, the economic dynamics conditioned, in a high degree, the scope of the discussion. Our analysis shows that the world of labor is an ideal place to observe the social construction of disease.
\end{abstract}

Keywords: Simulation. Psychiatry. Legal Medicine. Work accidents. Occupational Diseases. Social Security. 